\title{
Use of antibiotics in newborns with maternal history of premature rupture of membrane
}

\section{Manandhar SR', Manandhar DS ${ }^{2}$}

'Sunil Raja Manandhar, Assistant Professor; ${ }^{2}$ Dharma Sharna Manandhar, Former Professor and Head of Department of Paediatrics, Kathmandu Medical College Teaching Hospital, Kathmandu, Nepal

\begin{abstract}
Background: The premature rupture of membrane is a major risk factor for the early onset sepsis in neonates. It increases the risk of serious neonatal infection to one percent, compared to $0.5 \%$ for women with intact membrane. There is a conventional practice of using antibiotics in most of the babies with maternal history of premature rupture of membrane $>18$ hours. It is noticed that all the babies may not need prophylactic antibiotics in these conditions and there should be judicial use of antibiotics in babies with maternal history of premature rupture of membrane.

Objectives: To identify the risk factors for use of antibiotics in newborn babies born at Kathmandu Medical College Teaching Hospital with maternal history of premature rupture of membrane and to observe the outcome of the babies. Method: This is a prospective observational study done at Kathmandu Medical College Teaching Hospital. All newborns with maternal history of premature rupture of membrane for more than 18 hours were screened for neonatal sepsis and managed according to the treatment protocol. Ethical clearance was obtained and data were analyzed in statistical package for social science 16 .

Results: Among 160 cases included, 99 (62\%) mothers received antibiotics for premature rupture of membrane and only 54 (35\%) babies' required antibiotics. Among them 30 babies required oral antibiotics, 20 babies required injectable antibiotics and four babies required both antibiotics(oral and injectable), which is statistically significant ( $p$ value 0.03 ). Conclusion: This study has highlighted that every babies may not need antibiotics with maternal history of premature rupture of membrane. The use of antibiotics to mother for PROM before delivery has a significant beneficiary effect to babies reducing incidence of sepsis.
\end{abstract}

Key words: Neonatal sepsis, Postnatal antibiotic use, Premature rupture of membrane

\section{INTRODUCTION}

The premature rupture of membrane (PROM) is 1 a major risk factor for the early onset of sepsis in neonates. It increases the risk of serious neonatal infection to one percent, compared to $0.5 \%$ for women with intact membrane ${ }^{1}$. The mortality rate for neonatal sepsis is high, with range from $5 \%$ to $50 \%{ }^{2}$. PROM is defined as a rupture of amniotic membrane for $>18$ hours for Asian countries ${ }^{3}$. Preterm, pre-labor rupture of membranes occurs when the amniotic sac enclosing the fetus ruptures before 37 weeks of gestation and prior to the onset of labor (pre-labor) ${ }^{4}$. It is associated

Address for correspondence

Dr. Sunil Raja Manandhar

Assistant Professor

Department of Paediatrics

Kathmandu Medical College Teaching Hospital

Kathmandu, Nepal

E-mail: drsunilraja@gmail.com with about one-third of pre-term deliveries in highincome countries, and is associated with increased rate of neonatal and maternal infection ${ }^{5}$.

Early onset of bacterial infection is an important cause of morbidity and mortality in newborn infants. Various factors that increase the risk of neonatal infection have been identified. It is unclear whether newborn infants who are asymptomatic, born to mothers with one or more of these risk factors should receive prophylactic antibiotics rather than selectively if only clinical or microbiological evidence of sepsis emerges ${ }^{6}$.

Five potential maternal risk factors for sepsis are PROM for $>18$ hours maternal fever, chorioamnionitis, maternal cultures positive for Group B Streptococcus (GBS) prior to delivery and no administration of antibiotics to the mother during labor and delivery ${ }^{3}$. 
The two main sources of neonatal bacterial infection are the mother and the environment. Infections that appear in the first two days of life are usually the result of exposure to microorganisms of maternal origin? ${ }^{7}$. Risk factors for perinatally acquired bacterial infection in the neonate includes maternal fever during labor, preterm or premature rupture of membranes, preterm onset of labor, chorioamnionitis, maternal urinary tract infection, and maternal genital tract colonization with GBS at 3537 weeks of pregnancy ${ }^{8}$.

In case of PROM babies, the Centers for Disease Control (CDC) ${ }^{8}$ has not recommended routine use of antimicrobial prophylaxis for newborns whose mothers received intrapartum chemoprophylaxis for GBS infection, and further recommends that a healthy appearing infant whose mother received $>4$ hours of intrapartum prophylaxis may be discharged home after 24 hours if other discharge criteria have been met and a person able to comply fully with instructions for home observation is available. Lastly, the CDC recommends that if laboratory results and clinical course do not indicate bacterial infection, duration of therapy may be as short as 48 hours in the infant ${ }^{9}$.

Nevertheless, it is a common practice of giving antibiotics with history of PROM of $>18$ hours even with negative cultures, an unremarkable complete blood count (CBC), or with negative C-reactive protein in neonates who appear completely well. It appears that there is often substantial variation in practice for suspected sepsis among neonatologists in the same hospital, as well as among neonatologists in different regions of the country ${ }^{3}$.

As there is a conventional practice of using antibiotics in most of the babies with maternal history of PROM of $>18$ hours and it is assumed that all babies born with maternal history of PROM may not need prophylactic antibiotics and there should be judicial use of antibiotics in babies with maternal history of PROM. So, the objectives of this study were to identify the risk factors for use of antibiotics in newborn babies associated with history of PROM for $>18$ hours and to observe the outcome of babies till one month of age.

\section{METHODS}

This is a prospective observational study done at neonatal unit of Kathmandu Medical College Teaching Hospital
(KMCTH). Study period was of two years duration (July 2012 - August 2014). Newborns (both term and preterm) with maternal history of PROM for $>18$ hours delivered at KMCTH were included in this study. After 6 hours of birth, babies venous blood were investigated for total leukocyte count (TLC), differential count (DLC), absolute neutrophils count (ANC), hemoglobin $(\mathrm{Hb})$, micro erythrocyte sedimentation rate (ESR), c- reactive protein (CRP) and peripheral smear for band cells and toxic granules. In suspected case of pneumonia, chest x-ray was done and in suspected case of meningitis, lumber puncture (LP) was done. Cerebro spinal fluid (CSF) was examined for TLC, DLC, protein, sugar. Blood culture and sensitivity (CS) was obtained with $1 \mathrm{ml}$ venous blood obtained in Blood Culture and Sensitivity (C/S) vial. CSF was sent in every suspected case of meningitis. Urine routine and culture sensitivity (CS) was sent in case of suspected urinary tract infection (UTI). The samples were sent to laboratory within half hour of the procedure. Blood culture was incubated in Mackonky Agar. CRP was done by qualitative latex agglutination method and blood parameters TLC/ DC/ Hb were performed by coulter method. Out born deliveries, asphyxiated babies requiring resuscitation, hemodynamically unstable babies during birth were excluded from the study. All babies with maternal history of PROM were admitted in special care baby unit (SCBU) and kept for observation till all the reports were available.

The ethical clearance for this study was obtained from Institutional Review Committee of Kathmandu Medical College. Data were analyzed in SPSS 16 version with frequency and tabulation. Cross tabulation among maternal intake of antibiotics or not for PROM with respect to CRP of baby, condition of baby at birth, condition of baby during observation, antibiotics use in postnatal period and outcome of baby were analyzed. Pearson Chi - square test ( $x^{2}$ test) was applied and $p$ value $<0.05$ was considered to be significant. Risk factors and laboratory criteria for neonatal sepsis was mentioned in Box. 1. All the babies were managed as per the protocol given in Box 2.

\section{RESULTS}

Total 160 PROM cases were included in this 2 years period (July 2012 - Aug 2014). Out of which 105 (66\%) 


\section{Box 1: Risk factors, clinical features and laboratory criteria for neonatal sepsis:}

A. Maternal Risk Factors ${ }^{10}$

- Maternal history of PROM for $\geq 18$ hours

- Foul smelling liquor

- Spontaneous preterm labor $<37$ weeks

- Maternal Intrapartum fever $>38^{\circ} \mathrm{C}$

B. Neonatal Risk Factors ${ }^{10}$

- Prematurity

- $\quad$ Low birth weight (Birth weight $<2.5 \mathrm{~kg}$ )

- Low apgar score

C. Clinical Features ${ }^{11}$

- Respiratory distress (tachypnea, grunting, increase oxygen requirement)

- Apnea/cyanosis

- Unstable temperature - hyperthermia /hypothermia

- Cardiovascular disturbance (tachycardia /bradycardia, poor peripheral perfusion)

- Poor feeding

- Lethargy, irritability and seizures

D. Laboratory diagnostic criteria for neonatal sepsis ${ }^{11}$

I) Direct method: Isolation of microorganisms in blood or urine or CSF culture.

II) Indirect method: Septic screen consider to be positive if any two or more out of following five parameters are present:

1. Leucopenia (TLC $<5000 / \mathrm{mm} 3$ )

2. Neutropenia (ANC $<1800 / \mathrm{mm} 3$ )

3. Immature neutrophils to total neutrophils $(\mathrm{I} / \mathrm{T})$ ratio $:>0.2$

4. Micro ESR : $>15 \mathrm{~mm} 1^{\text {st }}$ hour

5. C- Reactive Protein (CRP) : positive

\section{Box 2: Treatment Protocol in management of PROM cases:}

1. If baby is clinically well (showing no clinical features of sepsis) and with normal blood parameters, antibiotic is not be started but the baby is kept in close observation in special care baby unit (SCBU). Well Baby is discharged when blood culture is negative and followed up at $1^{\text {st }}$ week, $2^{\text {nd }}$ week and 4th week of postnatal life.

2. If baby is clinically well (showing no clinical features of sepsis), but has CRP positive, oral cefpodoxime (3 $3^{\text {rd }}$ generation cephalosporin) is started till blood culture becomes available. If, blood culture is negative and baby is clinically fine, Cefpodoxime is continued for total 7 days. The baby is discharged after completion of 7 days and is followed up as per above protocol.

3. If baby is clinically unwell with showing clinical feature of sepsis as mentioned in Box 1, Ampicillin $(100 \mathrm{mg} / \mathrm{kg})$ and Amikacin ( $15 \mathrm{mg} / \mathrm{kg}$ ) is started parenterely. If blood culture is negative, injectable antibiotics is given for a total of 7 days. But, if blood culture is positive, appropriate antibiotics according to sensitivity pattern is given parenterely for a total of 10 days.

4. If any problem occurred after discharge, the baby is re-admitted in SCBU as per above protocol. 
were male and 55 (34\%) were female babies. Maternal characteristics were analyzed. Only 7 (4\%) mothers have history of fever during delivery, 10 (2\%) mothers have history of foul smelling liquor and only $2(1 \%)$ mothers have history of urinary tract infection. 99 (62\%) mothers were given antibiotics before delivery for PROM and 99 (62\%) mothers were primigravida which is depicted in Table 1.

Similarly, mean maternal age was 25.5 ( \pm 3.9) years, average duration of maternal history of PROM was 37.39 $( \pm 29.5)$ hours and average duration of antibiotics given to mother before delivery was $1.56( \pm 1.2)$ days which are depicted in Table 2.

Neonatal parameters with maternal history of PROM > 18 hours were analyzed. On admission in observational care, 146 (91\%) babies were normal and only 14 (9\%) babies were sick. Similarly during observational care, 146 (92\%) babies were normal and only 13 (8\%) newborns condition was deterioted. Among 160 babies enrolled, 154 (96\%) babies were normal and discharged where as 6 (4\%) babies expired during observational care. $66 \%$ (106) babies did not require any antibiotics and were discharged after seven days of postnatal age and the lab parameters were all within normal limit. 106 (66\%) babies did not require any antibiotics and only 54 (34\%) babies required antibiotics in postnatal period. Out of them, 30 (18\%) babies were treated with oral antibiotics (cefpodoxime), 4 (3\%) babies required both (oral and injectable) antibiotics and 13\% (20) babies were treated with injectable antibiotics only. During discharge from hospital, out of 160 babies, 96\% (154) were fine and 6 (4\%) babies were expired, depicted in Table 3. Among four expired babies, three babies were due to Meningitis and one baby died due to Septicemia.

Table 4 depicts relationship of maternal use of antibiotics and various neonatal parameters. Among 160 babies, $31 \%$ (50) babies have positive CRP. Among those 50 CRP positive babies, 26 babies mother received antibiotics before delivery and 24 babies mother did not received antibiotics ( $p$ value 0.08 ), which is depicted in serial no. 1 of table 4 . Out of 160 babies, 14 babies were clinically unwell during observational care in SCBU. Among those 14 unwell babies', 2 babies' mother received antibiotic for PROM and 7 babies' mother who did not receive antibiotic became lethargic. Similarly, 2 babies' mother received antibiotic and 3 babies whose mother did not receive antibiotic for PROM became tachypoenic ( $p=$ 0.02).
Cross tabulation relation between maternal antibiotics received for $P R O M$ with respect to antibiotics used in babies were analyzed. Out of 160 babies, 65\% (106) babies did not require antibiotic in postnatal period. Out of 106 babies (antibiotics not required during observational care), 72 babies mother received antibiotic for PROM and 34 babies mother did not received antibiotic. Total 54 (35\%) babies required antibiotic during observational care. Among them 30 babies required oral antibiotic, 20 babies required injectable antibiotic and 4 babies required both antibiotic (oral and injectable), which is statistically significant ( $p$ value 0.03 ) as shown in serial no. 3 of Table 4.

Cross tabulation relation between maternal antibiotics received for $\mathrm{PROM}$ with respect to neonatal condition during observational care is done. Out of $160,8 \%$ (13) babies' condition was deterioted during observational care at SCBU. Among 13 babies, 9 babies' mother did not received antibiotic for PROM, which is statistically significant ( $p$ value 0.04 ) as shown in serial no. 4 of Table 4. Similarly, relation between maternal high vaginal swab (HVS) and maternal antibiotic received for PROM were also analyzed. Among four cases of Klebsiella isolated in HVS, in three cases mother did not received antibiotics for PROM and only in one case, mother received antibiotics for PROM, shown in serial no. 5 of Table 4 however is statistically not significant ( $p$ value 0.18 ). Blood culture was positive only in four babies (2.4\%) whose mother did not received antibiotic for PROM, shown in serial no. 6 of Table 4, which is statistically insignificant ( $p$ value 0.08 ).

Table 5 showed prevalence of Low birth weight (LBW) which in this study was $28 \%$. Out of 160 babies, $25 \%$ (41) babies were preterm, 73\% (116) babies were term and $2 \%$ (3) babies were post term. Cross tabulation among risk factors with respect to use of antibiotics were analyzed. Among 54 babies who required antibiotics at postnatal period, $28 \%$ (15) babies were preterm and $37 \%$ (20) babies were LBW. Out of 10 babies' mother with history of foul smelling liquor, 7 babies required antibiotic at postnatal period. However these findings were statistically insignificant.

During one month follow up of 154 babies, at first week of life, only two babies were readmitted for dysentery and treated with cefotaxim and amikacin for 7 days and discharged. During follow up at $2^{\text {nd }}$ weeks and $4^{\text {th }}$ week of life these 154 babies became normal. 
Table 1: Maternal Characteristics

\begin{tabular}{lcc}
\hline Variables & Number $(\mathbf{n}=\mathbf{1 6 0})$ & Percentage (\%) \\
Maternal history of fever & 7 & 4 \\
Yes & 153 & 96 \\
No & & 2 \\
Maternal history of foul smelling liquor & 10 & 98 \\
Yes & 150 & 1 \\
No & & 99 \\
Maternal history of Urinary tract infection & 2 & \\
Yes & 158 & 62 \\
No & & 38 \\
Mother on antibiotics before delivery & 99 & 62 \\
Yes & 61 & 35 \\
No & & 3 \\
Gravida & 99 & 57 \\
Primi & 4 & \\
Multi & & \\
\hline
\end{tabular}

Table 2: Maternal Characteristics on different variables

\begin{tabular}{lcc}
\hline Variables & Mean $( \pm$ SD $)$ & Range \\
Maternal age & $25.5( \pm 3.9)$ & $(17-37$ years $)$ \\
Duration of PROM & $37.39( \pm 29.5)$ & $(18-192$ hours $)$ \\
\hline Maternal history of Antibiotics duration before delivery & $1.56( \pm 1.2)$ & $(1-10$ days $)$ \\
\hline
\end{tabular}

Table 3: Neonatal outcomes of babies with maternal history of premature rupture of membrane

\begin{tabular}{|c|c|c|}
\hline Variables & Number $(n=160)$ & Percentage (\%) \\
\hline \multicolumn{3}{|l|}{ Outcome of babies } \\
\hline Good & 154 & 96 \\
\hline Expired & 6 & 4 \\
\hline \multicolumn{3}{|c|}{ Condition of baby during Observational care } \\
\hline Normal & 146 & 91 \\
\hline Sick & 14 & 9 \\
\hline \multicolumn{3}{|l|}{ Use of antibiotics } \\
\hline No antibiotics & 106 & 65 \\
\hline Oral & 30 & 19 \\
\hline Injectable & 20 & 13 \\
\hline Oral and Injectable & 4 & 3 \\
\hline \multicolumn{3}{|c|}{ Condition deterioted during stay at hospital } \\
\hline Yes & 13 & 8 \\
\hline No & 147 & 92 \\
\hline
\end{tabular}


Table 4: Cross tabulation among maternal use of antibiotics with respect to different neonatal parameters

\begin{tabular}{|c|c|c|c|c|}
\hline \multirow{2}{*}{ Variables } & \multicolumn{2}{|c|}{ Mother on antibiotics for PROM } & \multirow{2}{*}{ Total } & \multirow{2}{*}{$\begin{array}{c}p \\
\text { value }\end{array}$} \\
\hline & Yes & No & & \\
\hline CRP & $\begin{array}{c}\text { Number of } \\
\text { babies }\end{array}$ & $\begin{array}{c}\text { Number of } \\
\text { babies }\end{array}$ & $\begin{array}{c}\text { Number } \\
\text { (percentage) }\end{array}$ & \multirow{4}{*}{0.08} \\
\hline Positive & 26 & 24 & $50(31)$ & \\
\hline Negative & 73 & 37 & $110(69)$ & \\
\hline Total & 99 & 61 & $160(100)$ & \\
\hline \multicolumn{5}{|c|}{ Neonatal condition during observational care } \\
\hline Normal & 95 & 51 & $146(91)$ & \multirow{4}{*}{0.02} \\
\hline Lethargic & 2 & 7 & $9(6)$ & \\
\hline Tachypoenic & 2 & 3 & $5(3)$ & \\
\hline Total & 99 & 61 & $160(100)$ & \\
\hline \multicolumn{5}{|l|}{ Use of antibiotics in babies } \\
\hline No antibiotics & 72 & 34 & $106(65)$ & \multirow{5}{*}{0.03} \\
\hline Oral & 19 & 11 & $30(19)$ & \\
\hline Injectable & 8 & 12 & $20(13)$ & \\
\hline Oral+ Injectable & 0 & 4 & $4(3)$ & \\
\hline Total & 99 & 61 & $160(100)$ & \\
\hline \multicolumn{5}{|c|}{ Neonatal condition deterioted during observational care } \\
\hline Yes & 4 & 9 & $13(8)$ & \multirow{3}{*}{0.04} \\
\hline No & 95 & 52 & $147(92)$ & \\
\hline Total & 99 & 61 & $160(100)$ & \\
\hline \multicolumn{5}{|l|}{ Maternal High Vaginal Swab CS } \\
\hline No growth & 96 & 57 & $153(96)$ & \multirow{5}{*}{0.14} \\
\hline Escherichia coli & - & 1 & $1(2)$ & \\
\hline Klebsiella & 1 & 3 & $4(3)$ & \\
\hline Normal flora & 2 & - & $2(1)$ & \\
\hline Total & 99 & 61 & $160(100)$ & \\
\hline \multicolumn{5}{|l|}{ Neonatal Blood CS } \\
\hline No growth & 98 & 58 & 156 ( 97.5) & \multirow{5}{*}{0.08} \\
\hline Coagulase negative Staphylococcus & - & 2 & $2(1.2)$ & \\
\hline Escherichia Coli & - & 1 & $1(0.6)$ & \\
\hline Staphylococcus Saprophyticus & - & 1 & $1(0.6)$ & \\
\hline Total & 98 & 62 & $160(100)$ & \\
\hline
\end{tabular}

Table 5: Cross tabulation among antibiotic use in babies with maternal history of PROM with respect to different risk factors

\begin{tabular}{|c|c|c|c|c|}
\hline \multirow{2}{*}{ Variables } & \multicolumn{2}{|c|}{ Babies on antibiotics for PROM } & \multirow[b]{2}{*}{ Total } & \multirow{2}{*}{ p value } \\
\hline & Yes & No & & \\
\hline Gestational age & Number of babies & Number of babies & $\begin{array}{c}\text { Number } \\
\text { (percentage) }\end{array}$ & \\
\hline $26-36.9$ wks (Preterm) & 15 & 26 & $41(25)$ & 0.73 \\
\hline $37-41.9$ wks (Term) & 39 & 77 & $116(73)$ & \\
\hline $42-44$ wks ( Post term) & - & 3 & $3(2)$ & \\
\hline \multicolumn{5}{|l|}{ Birth weight } \\
\hline $1-2.49 \mathrm{~kg}$ & 20 & 25 & $45(28)$ & \\
\hline $2.5-3.99 \mathrm{~kg}$ & 34 & 79 & $113(71)$ & 0.13 \\
\hline $4-5 \mathrm{~kg}$ & - & 2 & $2(1)$ & \\
\hline \multicolumn{5}{|c|}{ Maternal history of foul smelling liquor } \\
\hline Yes & 3 & 7 & $10(6)$ & \multirow{2}{*}{0.44} \\
\hline No & 103 & 47 & $150(94)$ & \\
\hline
\end{tabular}




\section{DISCUSSION}

As the current practice in our region, prophylactic antibiotic is used for babies born with maternal history of PROM. The result of various studies has suggested that the decision to use antibiotic in every baby with maternal history of PROM were not always justifiable. A study done by Jagjit ST et al have treated selectively to term babies with positive screening test with PROM in the absence of maternal symptoms of chorioamnionitis ${ }^{12}$. Similarly, in our study, $72 \%$ (72 out of 99 ) babies with maternal history of antibiotic intake and 55\% (34 out of 61) babies with maternal history of no antibiotic intake did not receive antibiotic in their postnatal period ( $p$ value 0.03 ) postulating that every babies with maternal history of PROM may not require antibiotic.

Another study done by Spitzer AR found that 286 infants (28.7\%) were treated with antibiotics for longer than 3 days in the overall population, even with negative antimicrobial cultures, full enteral feeding and stability in room air $^{3}$. Most of the babies with no maternal risk for sepsis have been treated as often those babies who have risk factors. Whereas in this study, $66 \%$ clinically well and sepsis negative babies (106 out of 160) did not receive any antibiotic and were discharged as normal baby suggesting that it is not necessary to treat all babies with maternal history of PROM and during follow up most of them are normal.

A study done by Kifah $A Q$ and Fatin Al found that the risk of neonatal infection with maternal history of antibiotic intake was $4.4 \%$ in babies compared with $11 \%$ in those whose mothers did not take antibiotics for PROM postulating that outcome of babies were better with maternal history of antibiotics intake than those mothers did not ${ }^{13}$. Similarly in this study also, we also found that the risk of neonatal infection was $4 \%$ (4 out of 99) with maternal history of antibiotic intake as compared to $16 \%$ (10 out of 61 ) with mothers who did not take antibiotic ( $p$ 0.02), highlighting that maternal history of antibiotics intake for PROM has a significant good impact on neonatal outcome.

Similarly, a study done by Shah GS et al at BP Koirala Institute of Health Sciences), Dharan highlighted that maternal history of PROM, maternal history of foul smelling liquor, prematurity, low birth weight and low Apgar score at birth were the strong risk factors for early onset neonatal sepsis ${ }^{10}$. In our study also prematurity, LBW and maternal history of foul smelling liquor were found to be significant risk factors for use of antibiotics in postnatal period.

\section{CONCLUSION}

Probably this is the first study done in Nepal highlighting the cautious use of antibiotics in babies with maternal history of premature rupture of membrane. This study has highlighted that every baby may not need antibiotic with maternal history of PROM (only $35 \%$ babies required antibiotic). The use of antibiotic in babies should be associated with risk factors like foul smelling liquor, prematurity, low birth weight, sick babies etc. The use of antibiotics in mother for PROM before delivery has a significant beneficiary effect to babies reducing incidence of sepsis. Further this type of study will help clinicians and pediatrician for judicial use of antibiotics in newborn baby with maternal history of PROM.

\section{ACKNOWLEDGEMENT}

Our sincere acknowledgment to all the Pediatric PG residents, medical officers for their contribution in data collection.

\section{REFERENCES}

1. Flannigan C, Hogan M. Prolonged rupture of membranes in term infants: Should all babies be screened? Clinical Audit. 2010 Feb; 2010(2):1-6.

2. Anderson-Berry A, Bellig L, Ohning B. Neonatal sepsis. E-Medicine. http://emedicine.medscape. com/article/978352-overview Updated: Oct 20, 2009.

3. Spitzer AR, Kirkby S and Kornhauser M. Practice Variation in Suspected Neonatal Sepsis: A Costly Problem in Neonatal Intensive Care. Journal of Perinatology 2005; 25:265-269.

4. Cousens S, Blencowe $\mathrm{H}$, Gravett $\mathrm{M}$ and Lawn JE. Antibiotics for pre-term pre-labour rupture of membranes: prevention of neonatal deaths due to complications of pre-term birth and infection. International Journal of Epidemiology 2010; 39:i134-i143.

5. Mercer B, Arheart K. Antimicrobial therapy in expectant management of preterm premature rupture of the membranes. Lancet 1995; 346:127179.

6. Ungerer RLS, Lincetto O, McGuire W, Saloojee H and Gulmezoglu AM. Prophylactic versus selective 
antibiotics for term newborn infants of mothers with risk factors for neonatal infection. Cochrane review. http://www.nichd.nih.gov/cochrane/ Ungerer/UNGERER.HTM.

7. World Health Organization. Perinatal Mortality: A listing of available information. WHO Report Geneva Switzerland; 2002.

8. Schuchat A, Wenger JD. Epidemiology of group B streptococcal disease. Risk factors, prevention strategies, and vaccine development. Epidemiologic Reviews 1994; 16: 374-402.

9. Verani JR. Prevention of Perinatal Group B Streptococcal Disease. Revised Guidelines from CDC. MMWR 2002; 51(RR11):1-22.
10. Shah GS, Budhathoki S, Das BK and Mandal RN. Risk factors in early neonatal sepsis. Kathmandu University Medical Journal (KUMJ). 2006 Apr-Jun; 4(2):187-91.

11. Ghai OP, Gupta P. Neonatal Sepsis. In: Gupta P, Paul VK and Bagga A (editors). Ghai Essential Pediatrics, 7th Ed. New Delhi (India): CBS Publications; 2010. pp $136-37$.

12. Jagjit ST, Srinivasan G, Rosita SP, Rydman RJ and Jacobs N. Management of asymptomatic neonates with prolonged rupture of membranes. Indian j Pediatr 1994; 61(1): 63-69.

13. Kifah $\mathrm{AQ}$ and Fatin Al. Neonatal outcome and prenatal antibiotic treatment in premature rupture of membranes. Pak J Med Sci 2005; 21(4):441-44. 